\title{
QOS based Approach for Web Service Recommendation Systems
}

\author{
Udhav Lahane ${ }^{1}$, K.N.Shedage ${ }^{2}$ \\ ${ }^{1,2}$ Department of Computer Engineering, Sir Visvesvaraya Institute of Technology, Nashik, Maharashtra, India
}

\begin{abstract}
A web service is a software system designed to support interoperable worldwide computer-to-computer interaction. Web services have been widely deployed for developing service-oriented applications in both industry and academia in recent years. The number of publicly available Web services is immovably increasing on the Internet. However, this generation makes it hard for a user to select a proper Web service among a large amount of service candidates. An unsuitable service selection may cause many problems (e.g., unsuitable performance) to the resulting applications. This paper, propose a novel CF-based Web service recommendation system for helping users select services with optimal Quality-ofService (QoS) performance. QoS (Quality-of-Service) is an important concept in cloud computing. It is very complicated to take decision on choosing the cloud services depending on QoS requirements. These requirements have to be fulfilled by both cloud service providers and cloud users. So, Excellent Service Selection is needed to obtain high quality cloud applications. With the increasing number of Cloud services, Quality-ofService (QoS) is usually employed for explaining non-functional properties of Cloud services. The QoS performance of cloud applications becomes low because of unreliable Internet connections. In this paper, we have presented a worldwide survey on QoS Ranking in Cloud Computing with respect to their Limitations and Inferences.
\end{abstract}

Keywords: Web service, Recommendation, Optimal Service Selection, Prediction, Quality-of-Service

\section{Introduction}

WEB services are software components designed to support interoperable machine-to-machine interaction over a network, usually the Internet. Web service employs WSDL (Web Service Description Language) for interface description and SOAP (Simple Object Access Protocol) for exchanging structured information. Benefiting from the cross-language and cross-platform characteristics, Web services have been widely employed by both enterprises and individual developers for building service-oriented applications. The adoption of Web services as a delivery model in business has fostered a paradigm shift from the development of monolithic applications to the dynamic set-up of business processes.

A web service enables communication among different applications by using open standards such as HTML, XML, WSDL, and SOAP. A web service get the help of:

- XML to attach the data

- SOAP to forword a message

A. Benefits of Web Services

Web services provide several technological and business benefits, a few of which include:

- Application and data integration

- Versatility

- Coder-use

- Cost savings

B. Quality-of-Service (QoS)

Quality-of-Service (QoS) is widely employed to represent the non-functional characteristics of Web services and has been considered as the key factor in service Selection. Quality of service (QoS) is the overall performance of a computer network, particular performance seen by users.

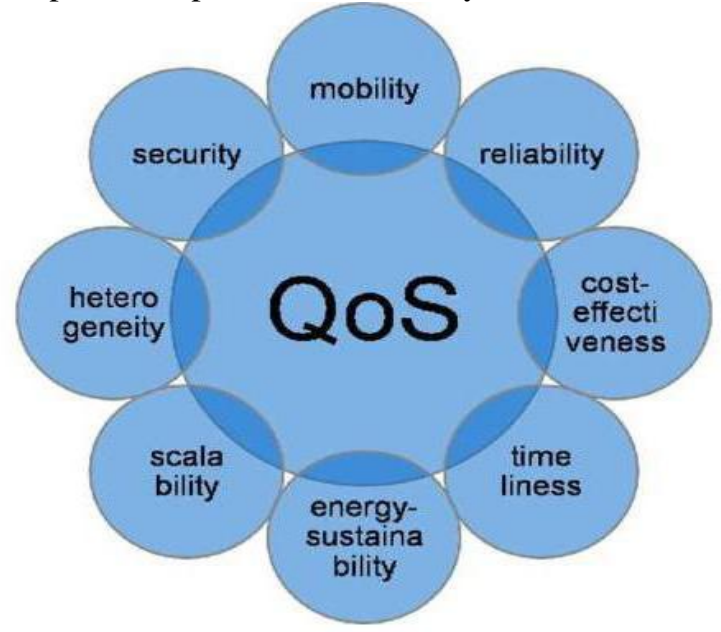

Figure 1: QoS Requirements for Web Services

With the generation of web services as a business solution to enterprise application integration, the QoS for web services is becoming progressively important to service providers. Quality of Service (QoS) of a web service is an important factor that differentiates similar services offered by different service provider. The QoS requirements for web services here mainly refer to the quality bearing of a web service. These may include reliability, performance, scalability, robustness, capacity, exception handling, integrity, accessibility, accuracy, availability, interoperability, and network-related QoS requirements. The enforcement of a web service represents how fast a service request can be completed. Web services should be provided with highest reliability. Reliability here explains the ability of a web service to perform its required functions under stated conditions for a specified time interval. 


\section{International Journal of Science and Research (IJSR) \\ ISSN (Online): 2319-7064 \\ Index Copernicus Value (2013): 6.14 | Impact Factor (2015): 6.391}

A web service [1] is a method of communication between two electronic devices all over the WWW. A web service is a software function provided at a network address over the web or the cloud, it is a service that is "always on" as in the concept of utility computing. "Web service" as software system designed to support interoperable machine-to-machine interaction over a network. It has an interface represents in a machine process able format (specifically WSDL). Other systems interact with the Web service in a manner prescribed by its description using SOAP messages, typically transmit using HTTP with an XML serialization in conjunction with other Web-related standards. Web services are distributed application peripherals that are externally available. You can use them to consolidate computer applications that are written in different languages and run on different platforms.

Web services are language and platform independent because merchant have agreed on common web service standards. Metro is a complete web services stack, covering all of a developer's needs from simple "Hello, World!" demonstrations to reliable, secured, and transacted web services. Metro added Web Services Interoperability Technologies (WSIT). WSIT supports enterprise features such as reliability, security and message optimization. WSIT make sure that Metro services with these features are interoperable with Microsoft .NET services. Within Metro, Project Tango develops and grows the code base for WSIT. Distinct programming models are available to web service developers. These models comes into two categories, both supported by the IDE: REST based. Representational State Transfer is a new idea to create and communicate with web services. In REST, resources have URIs and are employ through HTTP header operations. For more explanation, see Restful Web Services.

SOAP/WSDL based approach. In older web service models, web service interfaces are exposed through WSDL documents (a type of XML), which have URLs. Successive message exchange is in SOAP, another type of XML document. For more explanation, see SOAP-based Web Services. The remaining of the paper will be organized as follows: In section 2 , we see about the web services. In section 3 we discuss about WRS function. In 4, 5 and 6, we see about objective of our system, system design and algorithm respectively. In 7, we see about the disadvantages of the existing system and advantages of our proposed system. The conclusion of our paper is in this section 8 .

\section{Related Work}

Our proposed system utilizes and enhances the following techniques and approaches to endorse the quality of web services for developer's business needs.

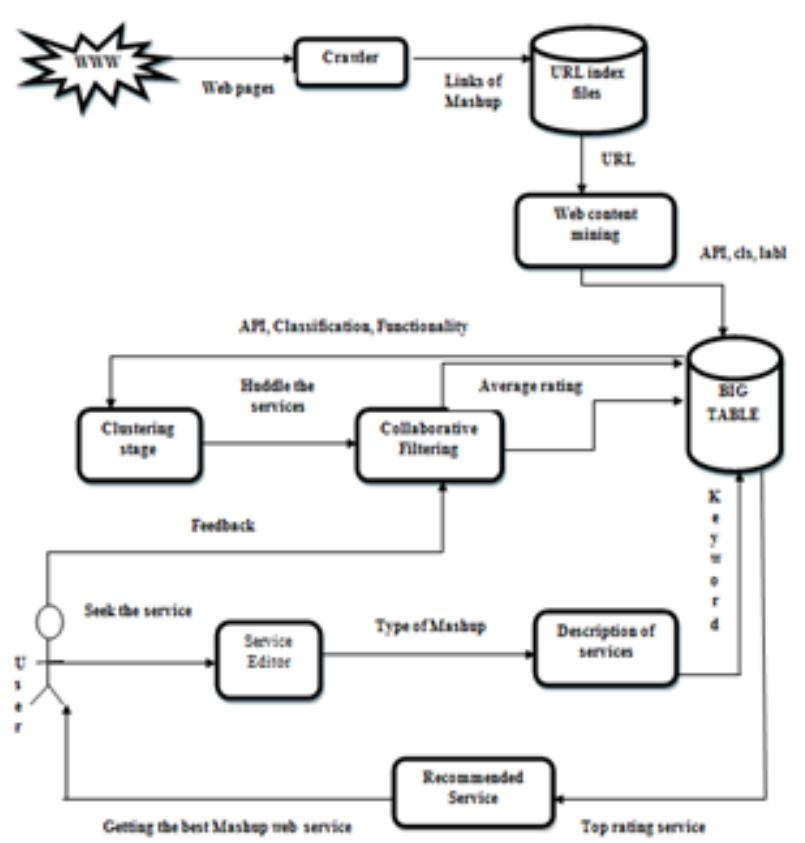

Figure 2: Architecture of background work.

Web Crawler is a simple program or automated script that scans through internet pages to create an index of web pages. We crawl all the publicly available Web services from World Wide Web that are all web service relevant web pages links are saved in to the database. Now, database contains the links of all the web service relevant web pages but each web page contains a lot of web pages that are hyperlinked inside a single web page then it also crawls each hyperlinked web page. The hyperlinked web page's links are also stored into the database.

Web Content Mining is used to extract the content of web services related Web page's links because of all the hyperlinked Web pages have superfluous text, images and also graphical data but we need only label of the web services, relevant APIs, classification, functionality from their links by using this web content mining technique. Web content mining is more divided into Web page content mining and search result mining. Web page content mining is traditional searching of Web pages with the help of content while search results mining is further search for pages found from previous search.

Clustering is the task of inventing homogenous group of data items. It is the partition of a set of data item into subsets. Data clustering is based on the similarity or dissimilarity counting between data items. In data mining, hierarchical clustering is the most important method of cluster which builds the hierarchy of clusters. Service users have difficulties to finding our desirable services because of tremendously increasing the service relevant data so we will group the similar services based on APIs, functionality, classification by using clustering algorithm. 


\section{International Journal of Science and Research (IJSR)}

ISSN (Online): 2319-7064

Index Copernicus Value (2013): 6.14 | Impact Factor (2015): 6.391

\section{Proposed Work}

The proposed system, try to propose personalized QoS value prediction for service users by employing the available past user experiences of Web services from different users. This approach requires no additional Web service invocations. Based on the anticipated QoS values of Web services, personalized QoS-aware Web service recommendations can be produced to help users select the optimal service among the functionally equivalent ones. From a huge number of currentworld service QoS data collected from number of locations, we found that the user focused Web service QoS performance has strong correlation to the locations of users. To enhance the prediction accuracy, system propose a location-aware Web service recommender system (named LoRec), which employs both Web service QoS values and user locations for making personalized QoS prediction. Users of LoRec share their past usage experience of Web services, and in return, the system provides personalized service recommendations to them. LoRec first collects user observed QoS records of different Web services and then groups users who have similar QoS observations together to generate recommendations. Location information is also considered when clustering services and users. The main contributions of this work are as follows:

- First, system proposes location-aware Web service recommendation approach, which significantly improves the recommendation accuracy.

- Second, system conduct comprehensive experiments to evaluate approach by employing a real-world Web service QoS data set.

\section{A. Web Service Recommendation}

Web service QoS prediction is used in different ways in LoRec to facilitate Web service recommendation. First, when a user searches Web services using LoRec, predicted QoS values will be shown next to each candidate service, and the one with the best predicted value will be highlighted in the search result for the active user. It will be easier for the active user to decide which one to have a try. Furthermore, LoRec selects the best performing services (services with the best submitted QoS) and services with the best predicted QoS from the whole service repository for the active user so that he/she can quickly find potential valuable ones instead of checking the service one by one.

\section{B. Advantages of Proposed System}

1) Improves the recommendation accuracy compared with existing service recommendation.

2) Web service recommender system help users to select services with optimal Quality-of-Service (QoS) performance.

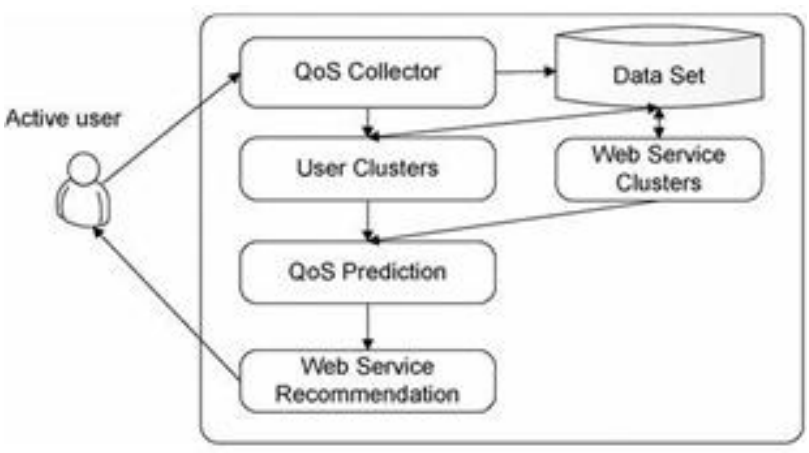

Figure 3: System Overview of LoRec

\section{System Design}

\subsection{System Architecture}

This paper is intended to provide an insight of the annotation techniques and application of few techniques to provide the required results with the above stated advantages. A collaborative filtering algorithm [8] designed for large-scale web service recommendation. This collaborative filtering algorithm uses a rule-based mechanism to determine behavior consistent information based control strategies for route guidance in a dynamic vehicular traffic system. This approach employs the characteristic of QoS and achieves considerable improvement on the recommendation accuracy.

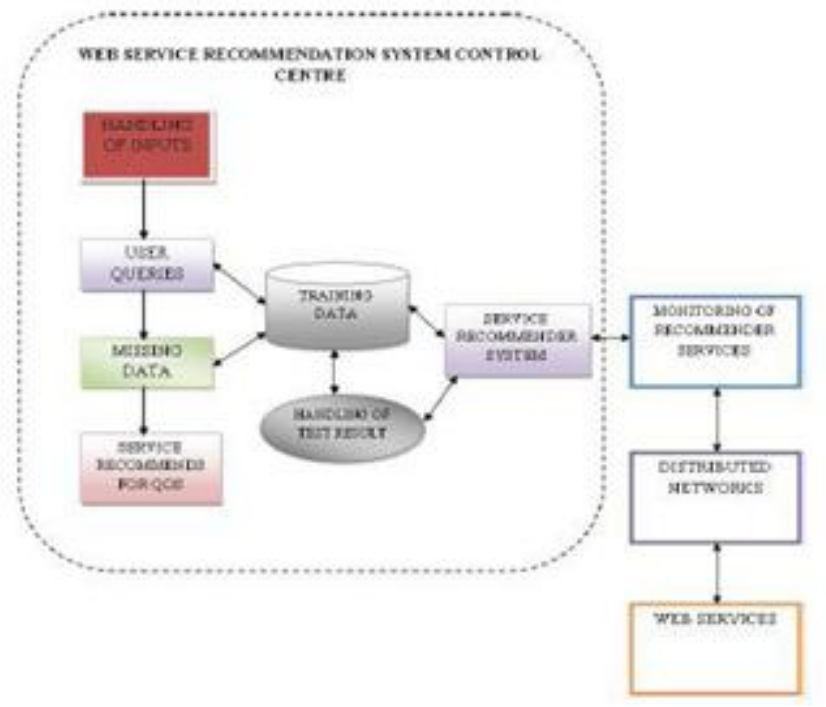

Figure 4: An Enhanced Web-service Recommendation

\subsection{System Architecture}

In the presence of multiple Web services with identical or similar functionalities, Quality of Service (QoS) provides nonfunctional Web service characteristics for the optimal Web service selection. Since the service providers may not deliver the QoS it declared, and some QoS properties (e.g. invocation failure-rate, network latency etc.) are highly related to the locations and network

Conditions of the service users, Web service evaluation by the service users can obtain more accurate results on whether the

Volume 5 Issue 6, June 2016 www.ijsr.net 


\section{International Journal of Science and Research (IJSR) \\ ISSN (Online): 2319-7064 \\ Index Copernicus Value (2013): 6.14 | Impact Factor (2015): 6.391}

demanded. The service recommender system component is answering the global user query. The latter has to be split local queries (i.e., sub-queries) and has to determine which peer is able to solve a local query. Each sub-query is expressed in SQL. SRS handles a Local Query Processing Engine component. Then, it carries out all the communication between the composed services and create a set of composition plans to give the requested data. The basic function of this architecture is to obtain sufficient Web service QoS information from different service users crucial for making accurate Web service recommendations. The idea is that by contributing the individually observe Web service QoS information to Web Service Recommender System, the service users can obtain accurate Web service recommendation service. Apart from the user contribution mechanism, Web Service Recommender System also controls a number of distributed computers for monitoring the publicly available Web services. The system architecture of Web Service Recommender System, which includes the following procedures: An active service user provides the individually obtained Web service QoS information to the Web Service Recommender System, The Input Handler in the Web Service Recommender System processes the input data, The Find Similar Users finds similar users from the training data of Web Service Recommender System, The Predict Missing Data predicts [17] the missing QoS values for the active user using collaborative filtering algorithm [10] and saves the predicted values and The Recommender employs the predicted QoS values to recommend optimal Web services to the active user.

\section{The Recommendation Approach}

\section{A. Motivating Scenario}

In this section, an online service searching scenario to show the research problem of this paper. The basic idea of this approach is that users closely located with each other are more likely to have similar service experience than those who live far away from each other. Changed by the success of Web 2.0 websites that emphasize collaboration, information sharing and interaction, we employ the idea of user-collaboration in our web service recommender system. The more QoS information the user contributes, the more proper service recommendations the user can obtain, since more user properties can be analyzed from the user contributed information. Based on the collected QoS records, our recommendation way is designed as a twoSteps process. In the first step, we divide the users into different regions based on their physical locations and older QoS experience on web services. In the second step, we find common users for the current user and make QoS anticipated for the unused services. Services with the best anticipated QoS will be recommended to the current user.

\section{B. Steps 1: Region Creation}

In web service recommender system, users usually provide QoS values on a small number of web services. Older memory-based CF algorithms suffer from the sparse user provided data set, since it ${ }^{\text {ee }} \mathrm{s}$ hard to find similar users without enough knowledge of their service experience. Different from present methods, we employ the correlation between users ${ }^{\text {ee }}$ physical locations and QoS properties to solve this problem. In this paper, we focus on the QoS properties that are prone to change and can be easily gained and objectively counted by individual users, such as response time and availability.

\section{Steps 2: QoS Value Prediction}

After the Steps of region aggregation, thousands of users are clustered into a certain number of regions based on their physical locations and historical QoS similarities. The service sense of users in a region is represented by the region center. With the compressed QoS data, searching neighbors and making predictions for an active user can be computed quickly. Traditionally, the QoS prediction methods need to search the entire data set, which is rather inefficient. In this approach, similarity between the active user and users of a region is computed by the similarity between the active user and the region center. Moreover, it is more reasonable to predict the QoS value for active users based on their regions, for users in the same region are more likely to have similar QoS experience on the same web service, especially on those region-sensitive ones.

\section{Conclusion}

This paper presents an innovative QoS-aware Web service recommendation approach . The basic idea is to predict Web services QoS values and recommend the best one for active users based on historical Web service QoS records. In order to better recommend Web services to users from amount of services with identical functions, this paper proposed a Web service recommendation approach based on collaborative filtering. Cloud computing aim is to provide scalable and adaptive to the diversity of end-users. Optimal service selection is important to obtain high quality cloud applications. A greedy algorithm treats rated and unrated items equally so it provides low quality cloud applications. Cloud Rank Framework provides the same quality in both algorithms. So, we suggest an optimal VM allocation is used to improve the quality of cloud applications. It also reduces the time complexity by providing an optimal value which ensures QoSAware Services. Our experimental result showed that our proposed novel technique works efficiently when compared to previous methods.

\section{References}

[1] M.B. Blake and M.F. Nowlan, "A Web Service

[2] Recommender System Using Enhanced Syntactical

[3] Matching," Proc. Int ${ }^{\circ} 1$ Conf. Web Services, pp. 575- 582, 2007.

[4] J.S. Breese, D. Heckerman, and C. Kadie, "Empirical

[5] Analysis of Predictive Algorithms for Collaborative

[6] Filtering," Proc. 14th Conf. Uncertainty in Artificial Intelligence (UAI "e98), pp. 43-52, 1998. 


\section{International Journal of Science and Research (IJSR) \\ ISSN (Online): 2319-7064}

Index Copernicus Value (2013): 6.14 | Impact Factor (2015): 6.391

[7] J. Himberg, "A SOM Based Cluster Visualization and Its Application for False Coloring," Proc. IEEEINNSENNS Int"1 Joint Conf. Neural Networks, pp. 587-592, 2000, vol. 3, doi:10.1109/IJCNN.2000.861379.

[8] Y. Pauline Jeba, "A survey on annotating search results from web databases," International journal of research in Computer Applications and Robotics.

[9] S. Wang,Q.Sun,andF. Yang,,,,Towards Web Service

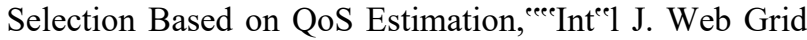
Serv.,vol.6,no.4, pp. 424-443, Nov. 2012.

[10] L. Shao, J. Zhang, Y. Wei, J. Zhao, B. Xie, and H. Mei,

[11] "Personalized QoS Prediction for Web Services via Collaborative Filtering," Proc. Int'1 Conf. Web Services, pp. 439-446, 2007.

[12] L. Shao, J. Zhang, Y. Wei, J. Zhao, B. Xie, and H. Mei, "Personalized QoS Prediction for Web Services via

[13] Collaborative Filtering," Proc. Int" 1 Conf. Web Services, pp. 439-446, 2007.

[14] M.R. McLaughlin and J.L. Herlocker, ,„,A Collaborative Filtering Algorithm and Evaluation Metric that Accurately Model the User Experience, ${ }^{\text {ece }}$ inProc. 27th Int"1 ACM SIGIR Conf. Res. Dev. Inf. Retrieval, 2004, pp. 329-336.

[15] B. Mehta, C. Niederee, A. Stewart, C. Muscogiuri, and E.J. Neuhold, "An Architecture for Recommendation Based Service Mediation," Semantics of a Networked World, vol. 3226, pp. 250- 262, 2004.

[16] M.R. McLaughlin and J.L. Herlocker, "A Collaborative Filtering Algorithm and Evaluation Metric That Accurately Model the User Experience," Proc. Ann. Int"1 ACM SIGIR Conf., pp. 329-336, 2004.

[17] J. Zhu, Y. Kang, Z. Zheng, and M.R. Lyu, ,,A Clustering-Based QoS Prediction Approach for Web Service Recommendation, ${ }^{\text {ece }}$ in Proc. 15th IEEE Int" ${ }^{\text {"e }}$ Symp. Obj./Compon./Serv.-Oriented Real-Time Distrib. Comput. Workshops, Apr. 2012, pp. 93-98

[18] C.D. Mining, P. Raghavan, and H. Schu“ tze, An Introduction to Information Retrieval. Cambridge Univ., 2009.

[19] J.E. Haddad, M. Manouvrier, and M. Rukoz, ,„,TQoS: Transac-tional and QoS-Aware Selection Algorithm for automatic Web Service Composition, ${ }^{e e c}$ IEEE Trans. Serv. Comput, vol.3, no.1, pp. 73-85, Jan/Mar. 2010.

[20] J. Herlocker, J. Konstan, A. Borchers, and J. Riedl „An Algorithmic Framework for Performing Collaborative Filtering, ${ }^{\text {eee }}$ in Proc. 22nd Int"1 ACM SIGIR Conf. Res. Dev. Inf. Retrieval, 1999, pp. 230- 237.

[21] R.M. Sreenath and M.P. Singh, ,„,Agent-Based Service Selection, ece J. Web Semantics, vol. 1, no. 3, pp. 261279, Apr. 2003. Fig. 6. Impact of. chen et al.: web service recommendation via exploiting location and qos information 1923.

\section{Author Profile}

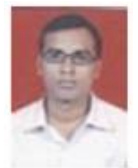

Udhav S. Lahane has completed his B.E in Information Technology from University of Pune in 2009. Currently pursuing Master of Engineering from SVIT Chincholi, Nashik, India.
Prof. K. N. Shedge is presently working as an Associate Professor in SVIT Chincholi, Nashik India

Volume 5 Issue 6, June 2016 www.ijsr.net 